\title{
ASYMPTOTIC ANALYSIS OF A GENERALIZED RICHARDSON EXTRAPOLATION PROCESS ON LINEAR SEQUENCES
}

\author{
AVRAM SIDI
}

\begin{abstract}
In this work, we give a detailed convergence and stability analysis for the author's generalized Richardson extrapolation process $\operatorname{GREP}^{(m)}$ as this is being applied to linearly convergent or divergent infinite sequences $\left\{A_{n}\right\}$, where $A_{n} \sim A+\sum_{k=1}^{m} \zeta_{k}^{n} \sum_{i=0}^{\infty} \beta_{k i} n^{\gamma_{k}-i}$ as $n \rightarrow \infty, \zeta_{k} \neq 1$ being distinct. The quantity we would like to compute is $A$, whether it is the limit or antilimit of $\left\{A_{n}\right\}$. Such sequences arise, for example, as partial sums of power series and of Fourier series of functions that have algebraic and/or logarithmic branch singularities. Specifically, we define the $\operatorname{GREP}^{(m)}$ approximation $A_{n}^{(m, j)}$ to $A$, with $n=\left(n_{1}, \ldots, n_{m}\right)$ and $\alpha>0$, via the linear systems

$$
A_{l}=A_{n}^{(m, j)}+\sum_{k=1}^{m} \zeta_{k}^{l} \sum_{i=0}^{n_{k}-1} \bar{\beta}_{k i}(\alpha+l)^{\gamma_{k}-i}, \quad j \leq l \leq j+\sum_{k=1}^{m} n_{k},
$$

where $\bar{\beta}_{k i}$ are additional unknowns. We study the convergence and stability properties of $A_{n}^{(m, j)}$ as $j \rightarrow \infty$. We show, in particular, that $A_{n}^{(m, j)}-$ $A=\sum_{k=1}^{m} O\left(\zeta_{k}^{j} j^{\gamma_{k}-2 n_{k}}\right)$ as $j \rightarrow \infty$. When compared with $A_{j}-A=$ $\sum_{k=1}^{m} O\left(\zeta_{k}^{j} j^{\gamma_{k}}\right)$ as $j \rightarrow \infty$, this result shows that $\operatorname{GREP}^{(m)}$ is a true convergence acceleration method for the sequences considered. In addition, we show that $\operatorname{GREP}^{(m)}$ is stable for the case being studied, and we also quantify its stability properties. The results of this work are the first ones pertaining to $\operatorname{GREP}^{(m)}$ with $m>1$.
\end{abstract}

\section{INTRODUCTION}

In [9], the author introduced a generalization of the Richardson extrapolation process and discussed some of its convergence and stability properties. This generalization - called $\operatorname{GREP}^{(m)}$ with $m$ a positive integer, or GREP for short has proved to be very useful in accelerating the convergence of a large class of infinite sequences with varying degrees of complexity in their behavior. Such sequences arise naturally in the summation of infinite series and in the computation of infinite-range integrals that may be oscillatory or monotonic, or that may behave in a more complicated manner. They also arise from trapezoidal rule approximations of finite-range simple or multiple integrals of regular or singular functions, etc. In addition, these sequences may be convergent or divergent. For an up-to-date treatment of GREP, its special cases, related algorithms, and its analytical theory, see the author's book [19, Chapters 4-13].

Received by the editor April 30, 2009 and, in revised form, June 25, 2009.

2000 Mathematics Subject Classification. Primary 40A05, 40A25, 41A60, 65B05, 65B10.

Key words and phrases. Acceleration of convergence, generalized Richardson extrapolation process, $\operatorname{GREP}^{(m)}$, power series, Fourier series, asymptotic expansions.

(C)2009 American Mathematical Society Reverts to public domain 28 years from publication 
The sequences for which $\operatorname{GREP}^{(m)}$ is useful arise from, and are identified with, functions $A(y)$ that belong to some general sets that were introduced in 9] and denoted there by $\mathbf{F}^{(m)}$. This set is defined as follows:

Definition 1.1. A function $A(y)$, defined for $0<y \leq b$, for some $b>0$, where $y$ can be a discrete or continuous variable, belongs to the set $\mathbf{F}^{(m)}$ if there exist functions $\phi_{k}(y)$ and $\beta_{k}(y), k=1, \ldots, m$, and a constant $A$, such that

$$
A(y)=A+\sum_{k=1}^{m} \phi_{k}(y) \beta_{k}(y)
$$

where the $\beta_{k}(\xi)$, as functions of the continuous variable $\xi$, are continuous in $[0, \widehat{\xi}]$ for some $\widehat{\xi} \leq b$, and have Poincaré-type asymptotic expansions (see, Olver [7], for example) of the form

$$
\beta_{k}(\xi) \sim \sum_{i=0}^{\infty} \beta_{k i} \xi^{i r_{k}} \quad \text { as } \xi \rightarrow 0+, \quad r_{k}>0, \quad k=1, \ldots, m .
$$

We assume that we know, or are able to compute, $A(y)$ only for $y>0$ but not for $y=0$. In case $\lim _{y \rightarrow 0+} \phi_{k}(y)=0, k=1, \ldots, m, \lim _{y \rightarrow 0+} A(y)$ exists and equals A. Otherwise, $\lim _{y \rightarrow 0+} A(y)$ does not exist, in which case we call $A$ the antilimit of $A(y)$. In many applications, the antilimit has a relevant meaning. We would like to compute $A$ whether it is the limit or antilimit of $A(y)$. This can be achieved in a very efficient way by $\operatorname{GREP}^{(m)}$, assuming that we know $A(y)$ along with $\phi_{k}(y)$ and $r_{k}, k=1, \ldots, m$. The definition of $\operatorname{GREP}^{(m)}$ is given next.

Definition 1.2. Let $A(y)$ belong to $\mathbf{F}^{(m)}$ with the notation of Definition 1.1 . Pick a decreasing positive sequence $\left\{y_{l}\right\} \subset(0, b]$ such that $\lim _{l \rightarrow \infty} y_{l}=0$. Let $n \equiv\left(n_{1}, n_{2}, \ldots, n_{m}\right)$, where $n_{1}, \ldots, n_{m}$ are nonnegative integers. Then, the approximation $A_{n}^{(m, j)}$ to $A$, whether $A$ is the limit or the antilimit of $A(y)$ as $y \rightarrow 0+$, is defined through the linear system

$$
A\left(y_{l}\right)=A_{n}^{(m, j)}+\sum_{k=1}^{m} \phi_{k}\left(y_{l}\right) \sum_{i=0}^{n_{k}-1} \bar{\beta}_{k i} y_{l}^{i r_{k}}, \quad j \leq l \leq j+N, \quad N=\sum_{k=1}^{m} n_{k},
$$

$\bar{\beta}_{k i}$ being the additional (auxiliary) $N$ unknowns. In (1.3), $\sum_{i=0}^{-1} c_{i} \equiv 0$ so that $A_{(0, \ldots, 0)}^{(m, j)}=A\left(y_{j}\right)$ for all $j$. This generalization of the Richardson extrapolation process that generates the $A_{n}^{(m, j)}$ is denoted $\left.\operatorname{GREP}^{(m)}\right]$

There are two different limiting processes concerning the $A_{n}^{(m, j)}$ : (i) that in which $n=\left(n_{1}, \ldots, n_{m}\right)$ is fixed and $j \rightarrow \infty$, called Process I, and (ii) that in which $j$ is fixed and $n_{k} \rightarrow \infty, k=1, \ldots, m$, simultaneously, called Process II. Of these, Process II is the one that is the most effective; therefore, we normally use the sequence $\left\{A_{(\nu, \ldots, \nu)}^{(m, j)}\right\}_{\nu=0}^{\infty}$ to approximate $A$.

Before we continue, we would like to note that, because $m \geq 1$ is an arbitrary integer, $\mathbf{F}^{(m)}$ includes most functions that arise in applications, hence is a very comprehensive set. Some special cases of $\operatorname{GREP}^{(m)}$ are the $D$ transformation for

\footnotetext{
${ }^{1}$ For the classical (polynomial) Richardson extrapolation process that covers Romberg integration among other applications, we have $m=1$ and $\phi_{1}(y)=y^{r_{1}}$, both in Definition 1.1 and in Definition 1.2 See Davis and Rabinowitz 3] Chapter 6], Stoer and Bulirsch [21, Chapter 3], or Sidi [19, Chapter 2], for example.
} 
computing infinite-range integrals and the $d$ transformation for summing infinite series, both of which are due to Levin and Sidi [5], and the $\bar{D}, \widetilde{D}, W$, and $m W$ transformations of the author [11, [12, [13], 117, for infinite-range (mostly oscillatory) integrals. The summation of power series and generalized Fourier series by the $d$ transformation is the subject of Sidi and Levin [20] and Sidi [14]. These transformations can also be used for computing multidimensional infinite-range integrals and for summing multidimensional infinite series; see Levin and Sidi [6].

The convergence and stability analysis of $\operatorname{GREP}^{(m)}$ with $m=1$ has been studied extensively by the author. The study in [15] and 18 concerns functions $A(y)$ that vary slowly (or behave smoothly) as $y \rightarrow 0$. The study in papers [10 14 mentioned above concerns functions $A(y)$ in $\mathbf{F}^{(1)}$ that vary quickly (e.g., behave exponentially or oscillate) as $y \rightarrow 0$. See also [19, Chapters 8 and 9]. So far, a detailed study of $\operatorname{GREP}^{(m)}$ with $m>1$, for functions $A(y)$ that are in $\mathbf{F}^{(m)}$ has not appeared in the literature, however. Such a study has proved to be exceedingly difficult because of the highly nonlinear nature of $\operatorname{GREP}^{(m)}$ with $m>1$.

The purpose of the present paper is to give the very first results pertaining to $\operatorname{GREP}^{(m)}$ with $m>1$ when $A(y)$ is in $\mathbf{F}^{(m)}$ and is varying quickly as $y \rightarrow 0$. Specifically, we consider sequences $\left\{A_{n}\right\}_{n=0}^{\infty}$ that are such that

$$
\begin{aligned}
& A_{n}=A+\sum_{k=1}^{m} \zeta_{k}^{n} B_{k}(n), \quad B_{k}(n) \sim \sum_{i=0}^{\infty} \beta_{k i} n^{\gamma_{k}-i} \text { as } n \rightarrow \infty, \\
& \zeta_{k} \neq 1 \text { distinct and arbitrary, } \quad \gamma_{k} \text { arbitrary. }
\end{aligned}
$$

Everything here can be real or complex. Comparing with Definition 1.1, we have the following analogy: $y=n^{-1}, A(y)=A_{n}, \phi_{k}(y)=\zeta_{k}^{n} n^{\gamma_{k}}, r_{k}=1, k=1, \ldots, m$. Of course, $y$ is necessarily discrete and takes on the values $1,1 / 2,1 / 3, \ldots$, only. Note that $\phi_{k}(y)$ are quickly varying because $\phi_{k}(y)=y_{k}^{\gamma} \exp \left[\left(\log \zeta_{k}\right) / y\right]$ and $\zeta_{k} \neq 1$. More specifically, (i) in case $\zeta_{k}$ is real and $0<\zeta_{k}<1, \phi_{k}(y) \rightarrow 0$ exponentially and monotonically as $y \rightarrow 0$, (ii) in case $\zeta_{k}$ is real and $-1<\zeta_{k}<0, \phi_{k}(y) \rightarrow$ 0 exponentially and oscillates about zero as $y \rightarrow 0$, and (iii) in case $\left|\zeta_{k}\right|=1$, hence $\zeta_{k}=e^{\mathrm{i} \theta_{k}}, \phi_{k}(y)=y^{\gamma_{k}} \exp \left(\mathrm{i} \theta_{k} / y\right)$ tends to zero or remains bounded or tends to infinity as $y \rightarrow 0$, depending on whether $\Re \gamma_{k}<0$ or $\Re \gamma_{k}=0$ or $\Re \gamma_{k}>0$, respectively; $\phi_{k}(y)$ oscillates about zero in all three cases.

To allow for more generality in the definition of $\operatorname{GREP}^{(m)}$, as it is applied in the next paragraph to the sequence $\left\{A_{n}\right\}$ described in (1.4), we introduce a parameter $\alpha>0$ that is at our disposal and reexpand the functions $B_{k}(n)$ in (1.4) as in

$$
A_{n}=A+\sum_{k=1}^{m} \zeta_{k}^{n} B_{k}(n), \quad B_{k}(n) \sim \sum_{i=0}^{\infty} \widehat{\beta}_{k i}(\alpha+n)^{\gamma_{k}-i} \quad \text { as } n \rightarrow \infty .^{2}
$$

Choosing $y_{l}=1 /(\alpha+l), l=0,1, \ldots$, and taking $n=\left(n_{1}, \ldots, n_{m}\right)$, where the $n_{i}$ are positive integers, we define $A_{n}^{(m, j)}$, the $\operatorname{GREP}^{(m)}$ approximation to $A$, along

\footnotetext{
${ }^{2}$ Note that both sequences $\left\{n^{\gamma_{k}-i}\right\}_{i=0}^{\infty}$ and $\left\{(\alpha+n)^{\gamma_{k}-i}\right\}_{i=0}^{\infty}$ are asymptotic scales as $n \rightarrow \infty$, and since $B_{k}(n)$ can be expanded in the former as in (1.4), it can be expanded in the latter as in (1.5) as well. The $\widehat{\beta}_{k i}$ in (1.5) are uniquely determined from the $\beta_{k i}$ in (1.4), and vice versa. For example, $\beta_{k 0}=\widehat{\beta}_{k 0}, \beta_{k 1}=\widehat{\beta}_{k 1}+\alpha \gamma_{k} \widehat{\beta}_{k 0}, \beta_{k 2}=\widehat{\beta}_{k 2}+\alpha\left(\gamma_{k}-1\right) \widehat{\beta}_{k 1}+\frac{1}{2} \alpha^{2} \gamma_{k}\left(\gamma_{k}-1\right) \widehat{\beta}_{k 0}$, and so on.
} 
with the auxiliary unknowns $\bar{\beta}_{k i}$, via the linear equations [cf. (1.3)]

$$
A_{l}=A_{n}^{(m, j)}+\sum_{k=1}^{m} \zeta_{k}^{l} \sum_{i=0}^{n_{k}-1} \bar{\beta}_{k i}(\alpha+l)^{\gamma_{k}-i}, \quad j \leq l \leq j+N, \quad N=\sum_{k=1}^{m} n_{k} .
$$

We know that (see Sidi [9] and [19, Theorem 4.2.2, p. 86]) $A_{n}^{(m, j)}$ can be expressed as in

$$
A_{n}^{(m, j)}=\sum_{i=0}^{N} \gamma_{n i}^{(m, j)} A_{j+i}
$$

for some scalars $\gamma_{n i}^{(m, j)}$ that satisfy

$$
\sum_{i=0}^{N} \gamma_{n i}^{(m, j)}=1
$$

The stability of the extrapolation process is ultimately connected with the quantity

$$
\Gamma_{n}^{(m, j)}=\sum_{i=0}^{N}\left|\gamma_{n i}^{(m, j)}\right|
$$

which determines the rate at which errors in the $A_{l}$ propagate into $A_{n}^{(m, j)}$. Note that, in view of (1.8), we have $\Gamma_{n}^{(m, j)} \geq 1$ always. We also know that the larger $\Gamma_{n}^{(m, j)}$ is, the less reliable the computed $A_{n}^{(m, j)}$ is. For a detailed discussion of stability in extrapolation, see [19, Section 0.5]. We will only mention that in case the $A_{l}$ are of approximately the same order of magnitude and are known with an accuracy of $t$ decimal digits, and $\Gamma_{n}^{(m, j)}$ is of order $10^{s}$, the computed and the exact $A_{n}^{(m, j)}$ will agree up to $t-s$ digits approximately; as a result, the computed $A_{n}^{(m, j)}$, as an approximation to $A$, will have at most $t-s$ correct digits.

In this work, we study the convergence and stability behavior of $A_{n}^{(m, j)}$ under Process I. Specifically, we analyze $A_{n}^{(m, j)}-A$ and $\Gamma_{n}^{(m, j)}$ as $j \rightarrow \infty$. To this effect, following some examples of sequences $\left\{A_{n}\right\}$ that satisfy (1.4) that we give in the next section, in Section 3, we give determinant representations of $A_{n}^{(m, j)}-A$ and of a polynomial related to the stability of $A_{n}^{(m, j)}$. Following that, in Section 4 , we state the main results concerning Process I, that is, as $j \rightarrow \infty$ while $n=\left(n_{1}, \ldots, n_{m}\right)$ is being held fixed. In Section [5] using the representations of Section 3, we give the proofs of the main results. In view of the main results of Section 4 , in Section 6 , we address the problems of slow convergence and reduced numerical stability in $\operatorname{GREP}^{(m)}$ that are present when $\zeta_{s} \approx 1$ in the complex plane for some $s$, and we justify the use of the so-called arithmetic progression sampling (APS) (see [19, Chapters $6,10,12,13])$ to remedy both problems.

Before proceeding further, we note that the computation of the sequence of approximations $\left\{A_{(\nu, \ldots, \nu)}^{(m, j)}\right\}_{\nu=0}^{\infty}$ can be done in a very efficient manner using the $W^{(m)}$ algorithm of Ford and Sidi [4]; see also [19, Chapter 7]. This algorithm is recursive in nature and does not necessitate the direct solution of the linear system in (1.6). The appendix to [4] contains a FORTRAN 77 program that implements the $d$ transformation using the $W^{(m)}$ algorithm. The $W^{(m)}$ algorithm is implemented via SUBROUTINE WMALGM in this program. The program appears also in the 
author's book [19, Appendix I], where GREP and all the different special cases mentioned earlier in this section are covered in great detail.

\section{EXAMPLES}

Sequences $\left\{A_{n}\right\}$ that behave as in (1.4) with $m=1$, that is,

$$
A_{n} \sim A+\zeta^{n} \sum_{i=0}^{\infty} \beta_{i} n^{\gamma-i} \text { as } n \rightarrow \infty, \quad \zeta \neq 1 \text { and } \gamma \text { arbitrary }
$$

are quite common and are said to be linearly convergent in case $\lim _{n \rightarrow \infty} A_{n}$ exists. $\lim _{n \rightarrow \infty} A_{n}$ exists if either (i) $|\zeta|<1$ or (ii) $|\zeta|=1$ and $\Re \gamma<0$. In case this limit exists, it is equal to $A$. Such sequences arise as partial sums of infinite series $\sum_{s=1}^{\infty} a_{s}$, namely, $A_{n}=\sum_{s=1}^{n} a_{s}, n=1,2, \ldots$, when $a_{n}$ satisfies

$$
a_{n} \sim \zeta^{n} \sum_{i=0}^{\infty} \alpha_{i} n^{\gamma-i} \text { as } n \rightarrow \infty, \quad \zeta \neq 1 \text { and } \gamma \text { arbitrary }
$$

See [19, Theorem 6.6.6, p. 145] for a proof of this statement.

In view of the above, it is clear that if $A_{n}=\sum_{s=1}^{n} a_{s}$, where

$$
\begin{aligned}
a_{n} & =\sum_{k=1}^{m} a_{n}^{(k)} ; \quad a_{n}^{(k)} \sim \zeta_{k}^{n} \sum_{i=0}^{\infty} \alpha_{k i} n^{\gamma_{k}-i} \text { as } n \rightarrow \infty, \\
\zeta_{k} & \neq 1 \text { distinct and arbitrary, } \quad \gamma_{k} \text { arbitrary, }
\end{aligned}
$$

then $A_{n}$ has an asymptotic expansion of the form given in (1.4). Here are two examples:

- Analytic functions that have $m$ algebraic branch points in the complex plane are one source of infinite series $\sum_{s=0}^{\infty} a_{s}$ that satisfy (2.3). For example, if $f(z)=c_{1} \log \left(1-\lambda_{1} z\right)+c_{2}\left(1+\lambda_{2} z\right)^{\omega}$, then $f(z)$ has the Maclaurin expansion

$$
f(z)=\sum_{n=0}^{\infty} a_{n}, \quad a_{0}=c_{2}, \quad a_{n}=-c_{1}\left(\lambda_{1} z\right)^{n} n^{-1}+c_{2}\left(\lambda_{2} z\right)^{n}\left(\begin{array}{c}
\omega \\
n
\end{array}\right), \quad n \geq 1 .
$$

Thus, $\zeta_{1}=\lambda_{1} z$ and $\gamma_{1}=-1$. From the fact that (see Abramowitz and Stegun [1, Eq. 6.1.47, p. 257])

$$
\begin{aligned}
\left(\begin{array}{c}
\omega \\
n
\end{array}\right) & =(-1)^{n} \frac{\Gamma(n-\omega)}{\Gamma(-\omega) \Gamma(n+1)} \\
& \sim(-1)^{n} \sum_{i=0}^{\infty} c_{i} n^{-\omega-1-i} \quad \text { as } n \rightarrow \infty, \quad c_{0}=\frac{1}{\Gamma(-\omega)},
\end{aligned}
$$

it follows that $\zeta_{2}=-\lambda_{2} z$ and $\gamma_{2}=-\omega-1$. Of course, $m=2$ in this case. Here $\Gamma(z)$ stands for the Gamma function.

- Another source of interest is the set of Fourier series of functions that have a number of algebraic singularities. To be more specific, let $f(x)$ be defined on $[-\pi, \pi]$, except at some points $x_{1}, x_{2}, \ldots$, in $[-\pi, \pi]$, where it has algebraic singularities of different strengths. Then its Fourier series is given by $\sum_{-\infty}^{\infty} c_{n} e^{\mathrm{i} n x}$, where $c_{n}=\frac{1}{2 \pi} \int_{-\pi}^{\pi} f(x) e^{-\mathrm{i} n x} d x$. The partial sums 
$\sum_{s=-n}^{n} c_{s} e^{\mathrm{i} s x}$ of this series are as in (1.4). As an example, let us consider

$$
f(x)=\left\{\begin{array}{lr}
0 & -\pi \leq x<a, \\
(x-a)^{\omega_{1}}(b-x)^{\omega_{2}} g(x) & a \leq x \leq b, \\
0 & b<x \leq \pi,
\end{array}\right.
$$

where $g \in C^{\infty}[a, b]$. That is, $f(x)$ has algebraic singularities at $x=a$ and $x=b$. For simplicity, let us assume that $f(x)$ is real so that twice the real part of the series $\frac{1}{2} c_{0}+\sum_{n=0}^{\infty} c_{n} e^{\mathrm{inx}}$ gives $f(x)$. It is known that (see, for example, Bleistein and Handelsman [2, Section 3.4])

$$
c_{n} \sim e^{-\mathrm{i} n a} \sum_{s=0}^{\infty} b_{1 s} n^{-\omega_{1}-1-s}+e^{-\mathrm{i} n b} \sum_{s=0}^{\infty} b_{2 s} n^{-\omega_{2}-1-s} \quad \text { as } n \rightarrow \infty .
$$

Thus, the partial sums $A_{n}=\frac{1}{2} c_{0}+\sum_{s=1}^{n} c_{s} e^{\mathrm{i} s x}$ are as in (1.4), with $m=2$ and $\zeta_{1}=e^{\mathrm{i}(x-a)}, \zeta_{2}=e^{\mathrm{i}(x-b)}, \gamma_{1}=-\omega_{1}-1$, and $\gamma_{2}=-\omega_{2}-1$.

\section{TECHNICAL PRELIMINARIES}

The results in the following lemma follow from [19, Section 4.2].

Lemma 3.1. Let $A_{n}^{(m, j)}$ be defined as in (1.6). For any scalars $g_{i}(j)$, define

$$
\left|g_{1}(j)\right| g_{2}(j)|\cdots| g_{p}(j) \mid=\left[\begin{array}{cccc}
g_{1}(j) & g_{2}(j) & \cdots & g_{p}(j) \\
g_{1}(j+1) & g_{2}(j+1) & \cdots & g_{p}(j+1) \\
\vdots & \vdots & & \vdots \\
g_{1}(j+N) & g_{2}(j+N) & \cdots & g_{p}(j+N)
\end{array}\right] .
$$

Clearly this is a matrix of dimension $(N+1) \times p$. With this notation, define the $(N+1) \times \nu$ matrix $D(\zeta, \gamma, \nu)$ by

$$
D(\zeta, \gamma, \nu)=\left|\zeta^{j}(\alpha+j)^{\gamma-\nu+1}\right| \zeta^{j}(\alpha+j)^{\gamma-\nu+2}|\cdots| \zeta^{j}(\alpha+j)^{\gamma-1}\left|\zeta^{j}(\alpha+j)^{\gamma}\right| \text {. }
$$

For an arbitrary sequence $\left\{x_{s}\right\}_{s=0}^{\infty}$, let $X_{j, N}=\left[x_{j}, x_{j+1}, \ldots, x_{j+N}\right]^{\mathrm{T}}$ and define also (3.3) $e_{n}^{(m, j)}\left(\left\{x_{s}\right\}\right)=\operatorname{det}\left[X_{j, N}\left|D\left(\zeta_{1}, \gamma_{1}, n_{1}\right)\right| D\left(\zeta_{2}, \gamma_{2}, n_{2}\right)|\cdots| D\left(\zeta_{m}, \gamma_{m}, n_{m}\right)\right]$.

[Note that the matrix on the right-hand side of (3.3) is $(N+1) \times(N+1)$.] Then, with $I_{s}=1$ for all $s$, we have

$$
A_{n}^{(m, j)}=\frac{e_{n}^{(m, j)}\left(\left\{A_{s}\right\}\right)}{e_{n}^{(m, j)}\left(\left\{I_{s}\right\}\right)}
$$

and

$$
\sum_{i=0}^{N} \gamma_{n i}^{(m, j)} z^{i}=\frac{e_{n}^{(m, j)}\left(\left\{z^{s}\right\}\right)}{e_{n}^{(m, j)}\left(\left\{I_{s}\right\}\right)} z^{-j} .
$$

The result in (3.4) can be obtained by applying Cramer's rule to the linear system in (1.6). The validity of the result in (3.5) [and also of that in (1.7) and (1.8)] can be shown by expanding the determinant $e_{n}^{(m, j)}\left(\left\{x_{s}\right\}\right)$ with respect to its first column and identifying $\gamma_{n i}^{(m, j)}$ as the cofactor of $x_{j+i}$ divided by $e_{n}^{(m, j)}\left(\left\{I_{s}\right\}\right)$.

The determinant representations of Lemma 3.1 will be very useful in our study later. 
Another tool that we will employ in our proofs and that concerns confluent Vandermonde determinants is given next. For a proof of this lemma, see Sidi [16, Eq. (3.15) and Appendix].

Lemma 3.2. Let the $(p+1) \times(p+1)$ matrix $H$ be given as in

$$
H=\left[H_{1}\left|H_{2}\right| \cdots \mid H_{t}\right]
$$

where

$$
H_{i}=\left[\begin{array}{ccccc}
1 & 0 & 0 & \cdots & 0 \\
c_{i} & c_{i} 1^{1} & c_{i} 1^{2} & \cdots & c_{i} 1^{s_{i}-1} \\
c_{i}^{2} & c_{i}^{2} 2^{1} & c_{i}^{2} 2^{2} & \cdots & c_{i}^{2} 2^{s_{i}-1} \\
\vdots & \vdots & \vdots & & \vdots \\
c_{i}^{p} & c_{i}^{p} p^{1} & c_{i}^{p} p^{2} & \cdots & c_{i}^{p} p^{s_{i}-1}
\end{array}\right], \quad i=1, \ldots, t ; \quad \sum_{i=1}^{t} s_{i}=p+1
$$

Then

$$
\operatorname{det} H=\left[\prod_{i=1}^{t}\left(\prod_{k=0}^{s_{i}-1} k !\right) c_{i}^{s_{i}\left(s_{i}-1\right) / 2}\right]\left[\prod_{1 \leq i<k \leq t}\left(c_{k}-c_{i}\right)^{s_{i} s_{k}}\right]
$$

\section{Main Results}

Our first result concerns the stability of Process I. It says that $\Gamma_{n}^{(m, j)}$ is bounded for all large $j$, which implies that Process $\mathrm{I}$ is stable.

Theorem 4.1. The polynomial $\sum_{i=0}^{N} \gamma_{n i}^{(m, j)} z^{i}$ satisfies

$$
\lim _{j \rightarrow \infty} \sum_{i=0}^{N} \gamma_{n i}^{(m, j)} z^{i}=\prod_{k=1}^{m}\left(\frac{z-\zeta_{k}}{1-\zeta_{k}}\right)^{n_{k}}
$$

Consequently,

$$
\lim _{j \rightarrow \infty} \Gamma_{n}^{(m, j)}=\lim _{j \rightarrow \infty} \sum_{i=0}^{N}\left|\gamma_{n i}^{(m, j)}\right| \leq \prod_{k=1}^{m}\left(\frac{1+\left|\zeta_{k}\right|}{\left|1-\zeta_{k}\right|}\right)^{n_{k}} .
$$

In case all the $\zeta_{k}$ are real positive or all are real negative, equality holds in (4.2). In case all the $\zeta_{k}$ are real negative, we thus have $\lim _{j \rightarrow \infty} \Gamma_{n}^{(m, j)}=1$.

Note that (4.1) implies that $\lim _{j \rightarrow \infty} \gamma_{n i}^{(m, j)}, i=0,1, \ldots, N$, all exist and are finite. In fact, they are the corresponding coefficients of the polynomial $\prod_{k=1}^{m}\left(\frac{z-\zeta_{k}}{1-\zeta_{k}}\right)^{n_{k}}$. Consequently, $\lim _{j \rightarrow \infty} \Gamma_{n}^{(m, j)}$ exists and is finite. This is part of (4.2), which also gives a very simple upper bound in terms of the $\zeta_{k}$.

The next theorem says that $\operatorname{GREP}^{(m)}$ accelerates the convergence of the sequence $\left\{A_{s}\right\}$ under Process I, providing at the same time the full asymptotic expansion of the error in $A_{n}^{(m, j)}$.

Theorem 4.2. Let us assume that $A_{n}$ is precisely as in (1.5) with the notation therein. Let us also define

$$
\chi_{n k i}^{(m, j)}=\frac{e_{n}^{(m, j)}\left(\left\{\zeta_{k}^{s}(\alpha+s)^{\gamma_{k}-i}\right\}\right)}{e_{n}^{(m, j)}\left(\left\{I_{s}\right\}\right)} .
$$

Then the following hold: 
(1) The $\chi_{n k i}^{(m, j)}$, for $i \geq n_{k}$, satisfy the asymptotic equalities

$$
\chi_{n k i}^{(m, j)} \sim\left(i-n_{k}+1\right)_{n_{k}}\left(\frac{\zeta_{k}}{\zeta_{k}-1}\right)^{n_{k}} \prod_{\substack{s=1 \\ s \neq k}}^{m}\left(\frac{\zeta_{s}-\zeta_{k}}{\zeta_{s}-1}\right)^{n_{s}} \zeta_{k}^{j} j^{\gamma_{k}-n_{k}-i} \quad \text { as } j \rightarrow \infty .
$$

Thus, the sequences $\left\{\chi_{n k i}^{(m, j)}\right\}_{i=n_{k}}^{\infty}$ are all asymptotic scales as $j \rightarrow \infty$; that $i s, \lim _{j \rightarrow \infty} \chi_{n k, i+1}^{(m, j)} / \chi_{n k i}^{(m, j)}=0$, for all $i \geq n_{k}$. Here $(a)_{s}=\prod_{p=1}^{s}(a+p-1)$ is the Pochhammer symbol.

(2) The error in the approximation $A_{n}^{(m, j)}$ has a genuine asymptotic expansion, given as in

$$
A_{n}^{(m, j)}-A \sim \sum_{k=1}^{m} \sum_{i=n_{k}}^{\infty} \widehat{\beta}_{k i} \chi_{n k i}^{(m, j)} \text { as } j \rightarrow \infty .
$$

(3) Let $\widehat{\beta}_{k, n_{k}+r_{k}}$ be the first nonzero $\widehat{\beta}_{k, n_{k}+s}$ with $s \geq 0$. Then

$$
A_{n}^{(m, j)}-A \sim \sum_{k=1}^{m} \zeta_{k}^{j} \sum_{i=0}^{\infty} \epsilon_{n k i} j^{\gamma_{k}-2 n_{k}-r_{k}-i} \quad \text { as } j \rightarrow \infty,
$$

where $\epsilon_{n k i}$ are some constants. Thus, we have, at worst,

$$
A_{n}^{(m, j)}-A=\sum_{k=1}^{m} O\left(\zeta_{k}^{j} j^{\gamma_{k}-2 n_{k}}\right) \quad \text { as } j \rightarrow \infty .
$$

Remark. (1) From the philosophy of the Richardson extrapolation process and its generalizations, we heuristically expect $\operatorname{GREP}^{(m)}$, as defined in (1.6), to "eliminate" the terms $\widehat{\beta}_{k i}(\alpha+j)^{\gamma_{k}-i}$, with $i=0,1, \ldots, n_{k}-1$, and $k=1, \ldots, m$, from the asymptotic expansion in (1.5), giving

$$
A_{n}^{(m, j)}-A=\sum_{k=1}^{m} O\left(\zeta_{k}^{j} j^{\gamma_{k}-n_{k}}\right) \quad \text { as } j \rightarrow \infty .
$$

The fact that Process I is stable as stated in Theorem 4.1 can be used to actually prove rigorously that $\operatorname{GREP}^{(m)}$, as defined in (1.6), does satisfy (4.8). For details, see [19, p. 89, Theorem 4.4.2]. Thus, the fact that we actually have a result such as that in (4.7) for the problem we are treating is quite surprising in view of the "expected" (4.8). [Note that (4.7) does not contradict (4.8); it is simply much stronger than (4.8).]

(2) The results of (4.4)-(4.6) are the best that can be obtained asymptotically under the given conditions. The simplicity of the leading term in the asymptotic expansion of $\chi_{n k i}^{(m, j)}$ given in (4.4) is also quite surprising.

(3) For $m=1$, Theorem 4.2 reduces to part (ii) of Theorem 19.2.3 in 19, p. 367], which was first given in Sidi [10].

\section{Proofs of MAIN Results}

For simplicity of notation, we will write

$$
w=(\zeta, \gamma, \nu) \text { and } \mu=\nu-1,
$$

for arbitrary $\zeta, \gamma$, and $\nu$. We will also write

$$
w_{k}=\left(\zeta_{k}, \gamma_{k}, n_{k}\right) \quad \text { and } \quad \mu_{k}=n_{k}-1, \quad k=1, \ldots, m .
$$


Thus, we will be using the short-hand notation

$$
D(w)=D(\zeta, \gamma, \nu) \quad \text { and } \quad D\left(w_{k}\right)=D\left(\zeta_{k}, \gamma_{k}, n_{k}\right), \quad k=1, \ldots, m
$$

throughout. We will use similar notation for all other matrices that depend on $\zeta_{k}$, $\gamma_{k}$, and $n_{k}$.

Finally, we will count the $N+1$ rows of all the matrices defined in Lemma 3.1 starting from $r=0$ up to $r=N$. Thus, for us, the $r$ th row of the matrix $G=$ $\left|g_{1}(j)\right| g_{2}(j)|\cdots| g_{p}(j) \mid$ in (3.1) is the vector

$$
G_{r}=\left[g_{1}(j+r), g_{2}(j+r), \ldots, g_{p}(j+r)\right], \quad r=0,1, \ldots, N .
$$

5.1. Proof of Theorem 4.1, We start with the analysis of the determinant $e_{n}^{(m, j)}\left(\left\{z^{s}\right\}\right)$. It is important to understand the details of the technique that we develop here because this technique is used again in the next subsection in the proof of Theorem 4.2. In this technique, we perform only elementary column transformations on $e_{n}^{(m, j)}\left(\left\{z^{s}\right\}\right)$. As we will see soon, it is sufficient to look at the matrix $D(\zeta, \gamma, \nu)$ given in (3.2) for this purpose. We recall that $D(\zeta, \gamma, \nu)=D(w)$ is $(N+1) \times \nu$.

Note that the $r$ th row of $D(w)$ is the vector

$$
\begin{aligned}
D_{r}(w)=\left[\zeta^{j+r}(\alpha+j+r)^{\gamma-\mu}, \zeta^{j+r}(\alpha+j+r)^{\gamma-\mu+1},\right. & \\
& \left.\ldots, \zeta^{j+r}(\alpha+j+r)^{\gamma-1}, \zeta^{j+r}(\alpha+j+r)^{\gamma}\right] .
\end{aligned}
$$

Factoring out the term $\zeta^{j}(\alpha+j)^{\gamma-\mu+i-1}$ from the $i$ th column of $D(w), i=1, \ldots, \nu$, we obtain the matrix $D^{\prime}(w)$ whose $r$ th row is

$$
D_{r}^{\prime}(w)=\left[\zeta^{r}\left(1+\epsilon_{r}\right)^{\gamma-\mu}, \zeta^{r}\left(1+\epsilon_{r}\right)^{\gamma-\mu+1}, \ldots, \zeta^{r}\left(1+\epsilon_{r}\right)^{\gamma-1}, \zeta^{r}\left(1+\epsilon_{r}\right)^{\gamma}\right]
$$

where

$$
\epsilon_{r}=r /(\alpha+j)
$$

The product of the terms we have factored out from the columns of $D(w)$ to obtain $D^{\prime}(w)$ is

$$
F_{1}(w ; j)=\zeta^{\nu j} \prod_{i=1}^{\nu}(\alpha+j)^{\gamma-\nu+i} .
$$

Let us now perform the following column transformations on the matrix $D^{\prime}(w)$ :

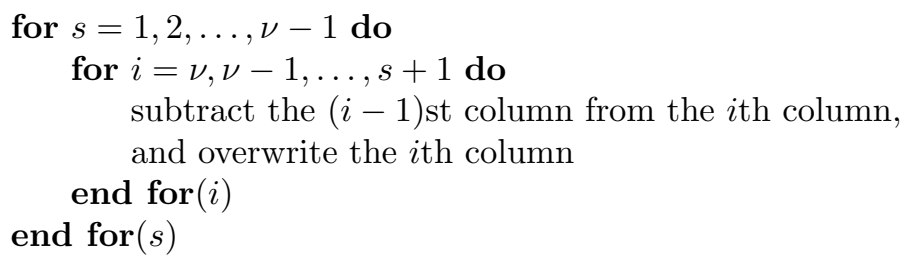

As a result of these transformations, we obtain the matrix $D^{\prime \prime}(w)$ whose $r$ th row is (5.7)

$D_{r}^{\prime \prime}(w)=\left[\zeta^{r}\left(1+\epsilon_{r}\right)^{\gamma-\mu} \epsilon_{r}^{0}, \zeta^{r}\left(1+\epsilon_{r}\right)^{\gamma-\mu} \epsilon_{r}^{1}, \ldots, \zeta^{r}\left(1+\epsilon_{r}\right)^{\gamma-\mu} \epsilon_{r}^{\mu-1}, \zeta^{r}\left(1+\epsilon_{r}\right)^{\gamma-\mu} \epsilon_{r}^{\mu}\right]$.

Factoring out the term $(\alpha+j)^{1-i}$ from the $i$ th column of $D^{\prime \prime}(w), i=1, \ldots, \nu$, and recalling (5.5), we obtain the matrix $D^{\prime \prime \prime}(w)$ whose $r$ th row is

$D_{r}^{\prime \prime \prime}(w)=\left[\zeta^{r}\left(1+\epsilon_{r}\right)^{\gamma-\mu} r^{0}, \zeta^{r}\left(1+\epsilon_{r}\right)^{\gamma-\mu} r^{1}, \ldots, \zeta^{r}\left(1+\epsilon_{r}\right)^{\gamma-\mu} r^{\mu-1}, \zeta^{r}\left(1+\epsilon_{r}\right)^{\gamma-\mu} r^{\mu}\right]$. 
The product of the terms we have factored out from the columns of $D^{\prime \prime}(w)$ to obtain $D^{\prime \prime \prime}(w)$ is

$$
F_{2}(w ; j)=\prod_{i=0}^{\mu}(\alpha+j)^{-i} .
$$

Summarizing, we have obtained from the matrix $D(w)$, by elementary column transformations, the matrix $D^{\prime \prime \prime}(w)$. The product of the terms we have factored out from the columns of $D(w)$ and $D^{\prime \prime}(w)$, until reaching $D^{\prime \prime \prime}(w)$, is

$$
F_{3}(w ; j)=F_{1}(w ; j) F_{2}(w ; j)=\left[\zeta^{j}(\alpha+j)^{\gamma-\mu}\right]^{\nu} .
$$

With these developments, also letting $Z^{0, N}=\left[z^{0}, z^{1}, \ldots, z^{N}\right]^{\mathrm{T}}$, we thus have

$$
\begin{aligned}
e_{n}^{(m, j)}\left(\left\{z^{s}\right\}\right)=z^{j}\left[\prod_{k=1}^{m} F_{3}\left(w_{k} ; j\right)\right] & \\
& \times \operatorname{det}\left[Z^{0, N}\left|D^{\prime \prime \prime}\left(w_{1}\right)\right| D^{\prime \prime \prime}\left(w_{2}\right)|\cdots| D^{\prime \prime \prime}\left(w_{m}\right)\right] .
\end{aligned}
$$

Now, as $j \rightarrow \infty$, we have that $\epsilon_{r} \rightarrow 0$; hence $\left(1+\epsilon_{r}\right)^{\omega} \sim 1$ for every $\omega$. Thus,

$$
D^{\prime \prime \prime}(w) \sim D^{\prime \prime \prime \prime}(w) \text { as } j \rightarrow \infty, \quad \text { entry-wise, }
$$

where the $r$ th row of $D^{\prime \prime \prime \prime}(w)$ is

$$
D_{r}^{\prime \prime \prime \prime}(w)=\left[\zeta^{r} r^{0}, \zeta^{r} r^{1}, \ldots, \zeta^{r} r^{\mu-1}, \zeta^{r} r^{\mu}\right]
$$

Consequently,

$$
\begin{aligned}
\operatorname{det}\left[Z^{0, N}\left|D^{\prime \prime \prime}\left(w_{1}\right)\right| D^{\prime \prime \prime}\left(w_{2}\right)|\cdots| D^{\prime \prime \prime}\left(w_{m}\right)\right] \sim \\
\operatorname{det}\left[Z^{0, N}\left|D^{\prime \prime \prime \prime}\left(w_{1}\right)\right| D^{\prime \prime \prime \prime}\left(w_{2}\right)|\cdots| D^{\prime \prime \prime \prime}\left(w_{m}\right)\right] \quad \text { as } j \rightarrow \infty,
\end{aligned}
$$

provided that the right-hand side is nonzero. By Lemma 3.2, the determinant on the right-hand side of (5.14) is given by

$$
\operatorname{det}\left[Z^{0, N}\left|D^{\prime \prime \prime \prime}\left(w_{1}\right)\right| D^{\prime \prime \prime \prime}\left(w_{2}\right)|\cdots| D^{\prime \prime \prime \prime}\left(w_{m}\right)\right]=K\left[\prod_{i=1}^{m}\left(\zeta_{i}-z\right)^{n_{i}}\right],
$$

where

$$
K=\left[\prod_{i=1}^{m}\left(\prod_{k=0}^{n_{i}-1} k !\right) \zeta_{i}^{n_{i}\left(n_{i}-1\right) / 2}\right]\left[\prod_{1 \leq i<k \leq m}\left(\zeta_{k}-\zeta_{i}\right)^{n_{i} n_{k}}\right]
$$

and is nonzero by the fact that the $\zeta_{k}$ are distinct. Combining everything, we finally have that

$$
e_{n}^{(m, j)}\left(\left\{z^{s}\right\}\right) \sim C(j) z^{j}\left[\prod_{i=1}^{m}\left(\zeta_{i}-z\right)^{n_{i}}\right] \quad \text { as } j \rightarrow \infty,
$$

where

$$
C(j)=K\left[\prod_{i=1}^{m} F_{3}\left(w_{i} ; j\right)\right] \neq 0 .
$$

Letting $z=1$ in (5.17), and recalling that $\zeta_{i} \neq 1$ for all $i$, we also have that

$$
e_{n}^{(m, j)}\left(\left\{I_{s}\right\}\right) \sim C(j)\left[\prod_{i=1}^{m}\left(\zeta_{i}-1\right)^{n_{i}}\right] \quad \text { as } j \rightarrow \infty .
$$


Because (5.17) and (5.19) are asymptotic equalities, we can divide the former by the latter and obtain the result in (4.1). The result in (4.2) is obtained by invoking [19, p. 31, Lemma 1.4.4].

5.2. Proof of Theorem 4.2, We start with the observation that the error in $A_{n}^{(m, j)}$ has a determinant representation given as in

$$
A_{n}^{(m, j)}-A=\frac{e_{n}^{(m, j)}\left(\left\{A_{s}-A\right\}\right)}{e_{n}^{(m, j)}\left(\left\{I_{s}\right\}\right)},
$$

which is easily obtained from (3.4). Substituting (1.5) in (5.20), and expanding the determinant $e_{n}^{(m, j)}\left(\left\{A_{s}-A\right\}\right)$ with respect to its first column, we obtain

$$
\begin{aligned}
A_{n}^{(m, j)}-A & =\frac{1}{e_{n}^{(m, j)}\left(\left\{I_{s}\right\}\right)} \sum_{k=1}^{m} e_{n}^{(m, j)}\left(\left\{\zeta_{k}^{s} B_{k}(s)\right\}\right) \\
& \sim \frac{1}{e_{n}^{(m, j)}\left(\left\{I_{s}\right\}\right)} \sum_{k=1}^{m} \sum_{i=0}^{\infty} \widehat{\beta}_{k i} e_{n}^{(m, j)}\left(\left\{\zeta_{k}^{s}(\alpha+s)^{\gamma_{k}-i}\right\}\right) \quad \text { as } j \rightarrow \infty
\end{aligned}
$$

Now, for $i=0,1, \ldots, n_{k}-1$, the determinant $e_{n}^{(m, j)}\left(\left\{\zeta_{k}^{s}(\alpha+s)^{\gamma_{k}-i}\right\}\right)$ is zero because it has two identical columns, namely, its first column and one of the columns in the matrix $D\left(w_{k}\right)$, which is part of $e_{n}^{(m, j)}\left(\left\{x_{s}\right\}\right)$ in 3.3). Consequently, the summation on $i$ in (5.21) begins with $i=n_{k}$. We have thus obtained the expansion in (4.5). Of course, in order for (4.5) to be a genuine asymptotic expansion, we must have

$$
\lim _{j \rightarrow \infty} \frac{\chi_{n k, i+1}^{(m, j)}}{\chi_{n k i}^{(m, j)}}=0, \quad i=n_{k}, n_{k}+1, \ldots, \quad k=1, \ldots, m .
$$

That (5.22) holds follows directly from (4.4), whose proof we tackle next.

By moving the first column of $e_{n}^{(m, j)}\left(\left\{\zeta_{k}^{s}(\alpha+s)^{\gamma_{k}-i}\right\}\right)$ to the right of the matrix $D\left(w_{k}\right)$, we obtain

$$
\begin{aligned}
& (-1)^{u_{k}} e_{n}^{(m, j)}\left(\left\{\zeta_{k}^{s}(\alpha+s)^{\gamma_{k}-i}\right\}\right) \\
& \quad=\operatorname{det}\left[D\left(w_{1}\right)|\cdots| D\left(w_{k-1}\right)\left|\widetilde{D}^{(i)}\left(w_{k}\right)\right| D\left(w_{k+1}\right)|\cdots| D\left(w_{m}\right)\right]
\end{aligned}
$$

where

$$
u_{k}=\sum_{i=1}^{k} n_{i}
$$

and $\widetilde{D}^{(i)}(w)$ is $(N+1) \times(\nu+1)$ and

$$
\begin{aligned}
& \widetilde{D}^{(i)}(w)=\left[D(w) \mid \widetilde{d}_{i}(w)\right], \\
& \widetilde{d}_{i}(w)=\left[\zeta^{j}(\alpha+j)^{\gamma-i}, \zeta^{j+1}(\alpha+j+1)^{\gamma-i}, \ldots, \zeta^{j+N}(\alpha+j+N)^{\gamma-i}\right]^{\mathrm{T}} .
\end{aligned}
$$

We first perform elementary column transformations on $D(w)$ to obtain $D^{\prime \prime}(w)$, precisely as in the preceding subsection. Next, we factor out the term $\zeta^{j}(\alpha+j)^{\gamma-i}$ from the last column $\widetilde{d}_{i}(w)$ of $\widetilde{D}^{(i)}(w)$ and obtain from $\widetilde{D}^{(i)}(w)$ the matrix $\widetilde{D}^{(i) \prime}(w)$ whose $r$ th row is

$$
\widetilde{D}_{r}^{(i) \prime}(w)=\left[D_{r}^{\prime \prime}(w), \zeta^{r}\left(1+\epsilon_{r}\right)^{\gamma-i}\right]
$$


We now rewrite the last entry of $\widetilde{D}_{r}^{(i) \prime}(w)$ in the form

$$
\zeta^{r}\left(1+\epsilon_{r}\right)^{\gamma-i}=\zeta^{r}\left(1+\epsilon_{r}\right)^{\gamma-\mu}\left(1+\epsilon_{r}\right)^{\mu-i}=\zeta^{r}\left(1+\epsilon_{r}\right)^{\gamma-\mu} \sum_{s=0}^{\infty}\left(\begin{array}{c}
\mu-i \\
s
\end{array}\right) \epsilon_{r}^{s} .
$$

From this and from (5.7), it is easy to see that, if we multiply the $(s+1)$ st column of $\widetilde{D}^{(i) \prime}(w)$ [hence the $(s+1)$ st column of $D^{\prime \prime}(w)$ ] by $\left(\begin{array}{c}\mu-i \\ s\end{array}\right), s=0,1, \ldots, \mu$, and subtract from the last column of $\widetilde{D}^{(i) \prime}(w)$, and overwrite the latter, we obtain the matrix $\widetilde{D}^{(i) \prime \prime}(w)$ whose $r$ th row is

$$
\begin{aligned}
\widetilde{D}_{r}^{(i) \prime \prime}(w) & =\left[D_{r}^{\prime \prime}(\zeta, \gamma), \zeta^{r}\left(1+\epsilon_{r}\right)^{\gamma-\mu} \sum_{s=\nu}^{\infty}\left(\begin{array}{c}
\mu-i \\
s
\end{array}\right) \epsilon_{r}^{s}\right] \\
& \left.=\left[D_{r}^{\prime \prime}(w), \zeta^{r}\left(1+\epsilon_{r}\right)^{\gamma-\mu} \epsilon_{r}^{\nu}\left\{\begin{array}{c}
\mu-i \\
\nu
\end{array}\right)+O\left(\epsilon_{r}\right)\right\}\right] .
\end{aligned}
$$

Factoring out the term $(\alpha+j)^{1-s}$ from the $s$ th column of $\widetilde{D}^{(i) \prime \prime}(w), s=1, \ldots, \nu$, and $\left(\begin{array}{c}\mu-i \\ \nu\end{array}\right)(\alpha+j)^{-\nu}$ from its last [that is, $(\nu+1)$ st] column, we obtain the matrix $\widetilde{D}^{(i) \prime \prime \prime}(w)$ whose $r$ th row is

$$
\widetilde{D}_{r}^{(i) \prime \prime \prime}(w)=\left[D_{r}^{\prime \prime \prime}(w), \zeta^{r}\left(1+\epsilon_{r}\right)^{\gamma-\mu} r^{\nu}\left\{1+\sum_{s=1}^{\infty} d_{r s}(\alpha+j)^{-s}\right\}\right],
$$

for some scalars $d_{r s}$.

With $\widetilde{D}^{(i)}\left(w_{k}\right)$ taken care of, we next perform on the matrices $D\left(w_{s}\right), s \neq k$, the exact same column transformations as in the preceding subsection.

With these developments, taking into account all the terms we factored out from all the columns of $e_{n}^{(m, j)}\left(\left\{\zeta_{k}^{s}(\alpha+s)^{\gamma_{k}-i}\right\}\right)$, including the $\left(n_{k}+1\right)$ st column of $\widetilde{D}^{(i)}\left(w_{k}\right)$, we have

$$
\begin{aligned}
& e_{n}^{(m, j)}\left(\left\{\zeta_{k}^{s}(\alpha+s)^{\gamma_{k}-i}\right\}\right)=E^{(i)}(j) \operatorname{det}\left[D^{\prime \prime \prime}\left(w_{1}\right) \mid \cdots\right. \\
& \left.\left|D^{\prime \prime \prime}\left(w_{k-1}\right)\right| \widetilde{D}^{(i) \prime \prime \prime}\left(w_{k}\right)\left|D^{\prime \prime \prime}\left(w_{k+1}\right)\right| \cdots \mid D^{\prime \prime \prime}\left(w_{m}\right)\right],
\end{aligned}
$$

where

$$
E^{(i)}(j)=(-1)^{u_{k}}\left(\begin{array}{c}
\mu_{k}-i \\
n_{k}
\end{array}\right) \zeta_{k}^{j}(\alpha+j)^{\gamma_{k}-n_{k}-i}\left[\prod_{s=1}^{m} F_{3}\left(w_{s} ; j\right)\right] .
$$

Going to the limit as $j \rightarrow \infty$ in $\widetilde{D}^{(i) \prime \prime \prime}(w)$, as before, we obtain the matrix $\widetilde{D}^{(i) \prime \prime \prime \prime}(w)$ whose $r$ th row is

$$
\widetilde{D}_{r}^{(i) \prime \prime \prime \prime}(w)=\left[D_{r}^{\prime \prime \prime \prime}(w), \zeta^{r} r^{\nu}\right]
$$

Combining everything in (5.23), we obtain

$$
\begin{aligned}
e_{n}^{(m, j)}\left(\left\{\zeta_{k}^{s}(\alpha+s)^{\gamma_{k}-i}\right\}\right) & \sim E^{(i)}(j) \operatorname{det}\left[D^{\prime \prime \prime \prime}\left(w_{1}\right) \mid \cdots\right. \\
& \left.\left|D^{\prime \prime \prime \prime}\left(w_{k-1}\right)\right| \widetilde{D}^{(i) \prime \prime \prime \prime}\left(w_{k}\right)\left|D^{\prime \prime \prime \prime}\left(w_{k+1}\right)\right| \cdots \mid D^{\prime \prime \prime \prime}\left(w_{m}\right)\right] \quad \text { as } j \rightarrow \infty,
\end{aligned}
$$

provided that the right-hand side of $(5.32)$ is nonzero. Note that $E^{(i)}(j) \neq 0$ for all $i \geq n_{k}$ because $\left(\begin{array}{c}\mu_{k}-i \\ n_{k}\end{array}\right) \neq 0$ by the fact that $\mu_{k}-i=n_{k}-i-1 \leq-1$. Again, applying Lemma 3.2, and rearranging, we have

$$
e_{n}^{(m, j)}\left(\left\{\zeta_{k}^{s}(\alpha+s)^{\gamma_{k}-i}\right\}\right) \sim \widetilde{C}^{(i)}(j)\left[\prod_{\substack{s=1 \\ s \neq k}}^{m}\left(\zeta_{s}-\zeta_{k}\right)^{n_{s}}\right] \quad \text { as } j \rightarrow \infty,
$$

where

$$
\widetilde{C}^{(i)}(j)=(-1)^{n_{k}}\left(n_{k} !\right)\left(\begin{array}{c}
\mu_{k}-i \\
n_{k}
\end{array}\right) \zeta_{k}^{j+n_{k}}(\alpha+j)^{\gamma_{k}-n_{k}-i} C(j) .
$$


Dividing the asymptotic equality in (5.33) by that in (5.19), and simplifying, we obtain the asymptotic equality in (4.4).

As for the full asymptotic expansion given in (4.6), we proceed as follows: In our analysis of the $\chi_{n k i}^{(m, j)}$, we were interested in finding only the leading-order term in its asymptotic expansion. With the term $\zeta_{k}^{j} j^{\gamma_{k}-n_{k}-i}$ factored out, it is easy to see from what remains from the quotient $e_{n}^{(m, j)}\left(\left\{\zeta_{k}^{s}(\alpha+s)^{\gamma_{k}-i}\right\}\right) / e_{n}^{(m, j)}\left(\left\{I_{s}\right\}\right)$ that an asymptotic expansion for it exists in negative powers of $j$. In other words, $\chi_{n k i}^{(m, j)}$ satisfies

$$
\chi_{n k i}^{(m, j)} \sim \zeta_{k}^{j} j^{\gamma_{k}-n_{k}-i} \sum_{s=0}^{\infty} a_{n k i s} j^{-s} \text { as } j \rightarrow \infty .
$$

Substituting (5.35) in (4.5), and rearranging, the result in (4.6) is obtained.

The rest of the proof is simple, and we leave it to the reader.

\section{Implications of Theorems 4.1 and 4.2}

In accelerating the convergence of linear sequences such as those treated in this work, one is confronted with serious convergence and numerical stability problems when $\zeta_{s} \approx 1$ for some $s$, irrespective of which method of acceleration is used. The presence of both of these problems when using GREP can be deduced from Theorems 4.1 and 4.2, thanks to the fact that both of these theorems provide the leading terms in the relevant asymptotic expansions.

Theorem 4.1 tells us that the $\gamma_{n i}^{(m, j)}$ are all, asymptotically as $j \rightarrow \infty$, proportional to $\prod_{k=1}^{m}\left(1-\zeta_{k}\right)^{-n_{k}}$. This immediately suggests that when $\zeta_{s}$, for some $s$, is very close to $1, \Gamma^{(m, j)}$ will be large (even though it remains bounded as $j \rightarrow \infty$ ); hence Process I will suffer from numerical stability problems in finite-precision arithmetic. That is, as $\zeta_{s}$ approaches 1, the numerical errors committed in the computation of $A_{n}^{(m, j)}$ will increase.

Similarly, (4.4) and (4.5) in Theorem 4.2 tell us that the error $A_{n}^{(m, j)}-A$ is, asymptotically as $j \rightarrow \infty$, proportional to $\prod_{k=1}^{m}\left(1-\zeta_{k}\right)^{-n_{k}}$. This immediately suggests that when $\zeta_{s}$, for some $s$, is very close to 1 , this error will be large; hence Process I will converge slowly, mathematically speaking. The convergence (and acceleration of convergence) of $A_{n}^{(m, j)}$ will slow down as $\zeta_{s}$ approaches 1 . This phenomenon has nothing to do with the computer arithmetic being used; it is purely theoretical.

Even though our results pertain to Process I, the problems of reduced numerical stability and slow convergence when $\zeta_{s} \approx 1$ for some $s$ are observed when GREP is applied under Process II as well.

To remedy this problem, it was proposed in [5] and 4] to apply GREP not to the whole sequence but to a subsequence $\left\{A_{\kappa n}\right\}$ of $\left\{A_{n}\right\}$ with some integer $\kappa \geq 1$. This choice of the subsequence has been called arithmetic progression sampling (APS for short) in [19, Chapter 10]. That this will solve both of the problems can be deduced from the fact that

$$
A_{\kappa n} \sim A+\sum_{k=1}^{m} \zeta_{k}^{\kappa n} \sum_{i=0}^{\infty} \beta_{k i}(\kappa n)^{\gamma_{k}-i} \quad \text { as } n \rightarrow \infty,
$$

which, defining

$$
\widetilde{A}_{n}=A_{\kappa n}, \quad \widetilde{\zeta}_{k}=\zeta_{k}^{\kappa}, \quad k=1, \ldots, m,
$$


can be rewritten as

$$
\widetilde{A}_{n} \sim A+\sum_{k=1}^{m} \widetilde{\zeta}_{k}^{n} \sum_{i=0}^{\infty} \widetilde{\beta}_{k i} n^{\gamma_{k}-i} \text { as } n \rightarrow \infty .
$$

Applying GREP to $\left\{\widetilde{A}_{n}\right\}$ (with $\zeta_{k}$ replaced by $\widetilde{\zeta}_{k}$, of course) we realize that Theorems 4.1 and 4.2 now hold with $\widetilde{\zeta}_{k}$ instead of $\zeta_{k}$. Whether $\left|\zeta_{s}\right| \leq 1$ or $\left|\zeta_{s}\right| \geq 1$, when $\zeta_{s}$ is close to 1 , we have that $\widetilde{\zeta}_{s}$ is farther from 1 than $\zeta_{s}$ is, even for $\kappa=2$. Therefore, by choosing $\kappa$ appropriately, we can cause $\widetilde{\zeta}_{s}$ to be away from 1 sufficiently to stabilize GREP numerically and to enhance its convergence.

An immediate application of this is to power series $\sum_{n=0}^{\infty} c_{n} z^{n}$, where

$$
c_{n} \sim \sum_{k=1}^{m} \sigma_{k}^{n} \sum_{i=0}^{\infty} \epsilon_{k i} n^{\gamma_{k}-i} \text { as } n \rightarrow \infty, \quad \sigma_{k} \neq 1 \text { distinct. }
$$

Then, the power series converges to a function $A(z)$ that is analytic for $|z|<$ $\min _{k}\left(1 /\left|\sigma_{k}\right|\right)=\rho$. In addition, letting $A_{n}(z)=\sum_{s=0}^{n} c_{s} z^{s}, n=0,1, \ldots$, we also have

$$
A_{n}(z) \sim A(z)+\sum_{k=1}^{m}\left(\sigma_{k} z\right)^{n} \sum_{i=0}^{\infty} \beta_{k i}(z) n^{\gamma_{k}-i} \text { as } n \rightarrow \infty .
$$

Clearly, $\zeta_{k}=\sigma_{k} z, k=1, \ldots, m$, in the notation of the preceding sections. In general, the function $A(z)$ can be continued analytically to $|z| \geq \rho, z_{k}=1 / \sigma_{k}$ being its branch points, with the branch cuts directed appropriately. [As an example, think of $A(z)$ as the sum of $m$ functions that have branch points at $m$ distinct points $z_{1}, \ldots, z_{m}$ in the $z$-plane.] Then $\zeta_{s} \approx 1$ means $z \approx z_{s}=1 / \sigma_{s}$, which in turn means that $z$ is near a point of singularity. Thus, we conclude that GREP will suffer from reduced numerical stability and from slow convergence when $z$ is close to a point of singularity of $A(z)$. Thus, close to points of singularity, applying GREP with APS will be very beneficial.

The topic of APS, within the context of the $d^{(m)}$ transformation (which is a $\operatorname{GREP}^{(m)}$, as mentioned in Section 1) is discussed in [19]. It is discussed rigorously in [19, Chapter 12, Section 12.7] for $m=1$, where a numerical example is also provided. In conjunction with the $d^{(m)}$ transformation for arbitrary $m \geq 1$, APS is applied to power series, Fourier series, and generalized Fourier series in [5, [14, [19, Chapter 6, Section 6.5 and Chapter 12, Section 12.9]. Its use in conjunction with the transformation of Shanks [8] is also proposed in [19, Chapter 16, subsection 16.5.1].

The results of the present work provide further theoretical justification for the use of APS within the context of $\operatorname{GREP}^{(m)}$ and the $d^{(m)}$ transformation, for all $m \geq 1$.

\section{REFERENCES}

[1] M. Abramowitz and I.A. Stegun, Handbook of mathematical functions with formulas, graphs, and mathematical tables, Nat. Bur. Standards Appl. Math. Series, no. 55, U.S. Government Printing Office, Washington, D.C., 1964. MR0167642 (29:4914)

[2] N. Bleistein and R.A. Handelsman, Asymptotic expansions of integrals, Holt, Rinehart and Winston, New York, 1975.

[3] P.J. Davis and P. Rabinowitz, Methods of numerical integration, second ed., Academic Press, New York, 1984. MR760629 (86d:65004)

[4] W.F. Ford and A. Sidi, An algorithm for a generalization of the Richardson extrapolation process, SIAM J. Numer. Anal. 24 (1987), 1212-1232. MR909075 (89a:65006) 
[5] D. Levin and A. Sidi, Two new classes of nonlinear transformations for accelerating the convergence of infinite integrals and series, Appl. Math. Comp. 9 (1981), 175-215, Originally appeared as a Tel Aviv University preprint in 1975. MR650681 (83d:65010)

[6] __ Extrapolation methods for infinite multiple series and integrals, J. Comput. Meth. Sci. Engrg. 1 (2001), 167-184.

[7] F.W.J. Olver, Asymptotics and special functions, Academic Press, New York, 1974. MR0435697 (55:8655)

[8] D. Shanks, Nonlinear transformations of divergent and slowly convergent sequences, J. Math. and Phys. 34 (1955), 1-42. MR0068901(16:961e)

[9] A. Sidi, Some properties of a generalization of the Richardson extrapolation process, J. Inst. Math. Appl. 24 (1979), 327-346. MR.550478 (81a:65011)

[10] _ Analysis of convergence of the T-transformation for power series, Math. Comp. 35 (1980), 833-850. MR572860 (83d:41039)

[11] _ Extrapolation methods for oscillatory infinite integrals, J. Inst. Math. Appl. 26 (1980), 1-20. MR.594340 (81m:40002)

[12] _ The numerical evaluation of very oscillatory infinite integrals by extrapolation, Math. Comp. 38 (1982), 517-529. MR645667 (83i:65023)

[13] _ A user-friendly extrapolation method for oscillatory infinite integrals, Math. Comp. 51 (1988), 249-266. MR942153 (89e:65029)

[14] Acceleration of convergence of (generalized) Fourier series by the d-transformation, Annals Numer. Math. 2 (1995), 381-406. MR1343544 (96h:65005)

[15] _ Convergence analysis for a generalized Richardson extrapolation process with an application to the $d^{(1)}$-transformation on convergent and divergent logarithmic sequences, Math. Comp. 64 (1995), 1627-1657. MR.1312099 (96a:65009)

[16] _ Further results on convergence and stability of a generalization of the Richardson extrapolation process, BIT Numerical Mathematics 36 (1996), 143-157. MR 1431580 (98f:65006)

[17] Computation of infinite integrals involving Bessel functions of arbitrary order by the $\bar{D}$-transformation, J. Comp. Appl. Math. 78 (1997), 125-130. MR1436784 (97k:65058)

[18] _ Further convergence and stability results for the generalized Richardson extrapolation process $G R E P^{(1)}$ with an application to the $D^{(1)}$-transformation for infinite integrals, J. Comp. Appl. Math. 112 (1999), 269-290. MR.1728465 (2000k:65045)

[19] _ Practical extrapolation methods: Theory and applications, Cambridge Monographs on Applied and Computational Mathematics, no. 10, Cambridge University Press, Cambridge, 2003. MR.1994507(2004e:65005)

[20] A. Sidi and D. Levin, Rational approximations from the d-transformation, IMA J. Numer. Anal. 2 (1982), 153-167. MR668590 (83j:65012)

[21] J. Stoer and R. Bulirsch, Introduction to numerical analysis, third ed., Springer-Verlag, New York, 2002. MR.1923481(2003d:65001)

Computer Science Department, Technion, Israel Institute of Technology, Haifa 32000, ISRAEL

E-mail address: asidi@cs.technion.ac.il

$U R L:$ http://www.cs.technion.ac.il/ ^asidi/ 